\title{
Feedback from the Sixth International AIDS Conference, San Francisco 1990
}

\begin{abstract}
Introduction
Following the Sixth International AIDS Conference in San Francisco in June 1990 a feedback day for genitourinary medicine physicians was held at University College London. This is a report from the feedback. Some of the abstracts have been published in full and these are referenced. The remainer of the abstracts are cited in the text using the code number from the published abstract books. A separate conference entitled Neurological and Neurophyschological Complications of HIV Infection was held in Monterey, California in the week before the main AIDS conference, and two of the contributors have included abstracts from Monterey.
\end{abstract}

\section{Epidemiology-Anne Johnson}

The 1990 International conference on AIDS in San Francisco brought compelling evidence of the continuing rapid and worldwide spread of HIV, wherever the behaviours occur which promote transmission. This is particularly apparent in areas previously relatively spared by the epidemic.

In South East Asia there is evidence of rapid spread, due primarily to prostitutes and to injecting drug users. Thailand is a striking example (FC99). A national serosurvey in 1989 found a considerable epidemic amongst injecting drug users, with between 33 and $50 \%$ infected in 10 provinces. Amongst prostitutes a survey of 2.5 thousand found that up to $44 \%$ have become infected in the course of just two or three years. As in Africa, the disease is emerging in STD clinic attenders, with $10 \% \mathrm{HIV}$ positive in the most severely affected province, $1 \%$ infected in antenatal clinic attenders, and already $0.91-3.7 \%$ in blood donors in the highest affected areas.

India is similarly affected. In Bombay, testing of 2,000 prostitutes showed evidence of spread occurring at $10 \%$ incidence per annum (FE612). Only $1.5 \%$ of prostitutes were infected in March 1988, and $23 \%$ in January 1990 . Similarly, in Madras there is evidence of increasing prevalence in prostitutes and STD clinic attenders (FC613). This pattern is similar to that seen a few years ago in cities in Africa.

There is increasing spread of HIV-1 to West Africa, particularly amongst travellers to and from high prevalence areas. HIV is, and increasingly will be, a major cause of mortality in Africa. In some African countries, screening blood donors has now been introduced. For example in Zambia up to $16 \%$ of donors are infected (FC609). This has lead to some discussion of the importance of nosocomial transmis- sion, (that is, transmission within hospitals and transmission via blood transfusion) which may have been further fuelling the sexual epidemic in Africa. The importance of nosocomial transmission has been enlightened by evidence emerging from Eastern Europe, where there appears to be a problem with transmission within hospitals. The Romanians have now reported 670 cases of AIDS of which 617 are children less than 4 years of age. Risk factors for infection have been microtransfusions of blood, (using 1 unit of blood for several children) the re-use of injecting equipment and poor sterilisation techniques due to electricity cuts in the middle of sterilising. The USSR has done some extensive epidemiological investigations. Of $40,000,000$ citizens tested 402 were found positive. About half of these have been infected by contaminated medical syringes, and blood transfusions (FC648). A particularly serious problem was identified in the rural area of Elista where several hundred people have been found to be infected through blood transfusion (FB462). In the USSR, therefore, the onward transmission is likely to be predominantly heterosexual.

Increasing evidence of heterosexual transmission of HIV was a recurring theme of the conference. In the Caribbean and South America, there is evidence of a change from a primary pattern I epidemiology (affecting homosexual men and drug users) to an increasingly heterosexually acquired epidemic (pattern II). Heterosexual spread is related to male bisexuality, injecting drug users and the emergence of HIV amongst female prostitutes (ThC710). For example in Brazil, $80 \%$ of cases of AIDS occurred in homosexual men in 1985 . This proportion has now dropped to only $52 \%$, with the male to female ratio falling from 120:1 to only 8:1 (FC98).

There is now some evidence that non-ulcerative STDs may have a role as co-factors in increasing the efficiency of HIV transmission. Marie Laga looked at 493 seronegative prostitutes in Kinshasa and followed them for 11 months (ThC97). Of these, 42 seroconverted and these were compared with 93 randomly selected controls. All the prostitutes had been examined for STDs longitudinally. Thus it was possible to examine the STDs that had been diagnosed during the window period, between five months and two months prior to seroconversion, and to compare the controls over the same time interval. Non-ulcerative STDs were more common in the seroconverters, such that purulent cervicitis occurred in $24 \%$ compared with $10 \%$ of controls; 
leukocytes in vaginal smears, $31 \%$ compared with $9 \%$; and trichomonas $43 \%$ compared with $18 \%$, suggesting that these were risk factors in increasing susceptibility amongst the prostitutes. They found that there was no difference between the seroconverters and non-seroconverters in numbers of contacts or condom use. Interestingly no difference was found in genital ulcer disease between the two groups $(4 \%$ compared with $8 \%$ ) in a population which has a low prevalence of genital ulcer disease. The implication for clinical practice is that control of non-ulcerative STDs may be as important in controlling HIV spread as control of ulcerative diseases.

Previous published studies have suggested that lack of circumcision in the male may increase the possibility of female to male transmission. This risk was studied in 357 men in a Kampala STD clinic of whom $42 \%$ were HIV antibody positive. $10 \%$ of the HIV positives had been circumcised compared with $17 \%$ of HIV negatives, giving an Odds Ratio of 0.54 $(0 \cdot 3-1 \cdot 0)$, suggesting that circumcision may be, to some extent, protective for female to male transmission (ThC577).

A further question which is frequently discussed is that of the relative efficacy of female to male as compared with male to female sexual transmission. A number of partner studies of female to male transmission were presented at the meeting. Studies presented at the meeting found between $24 \%$ and $13 \%$ of male partners of HIV positive females to be infected (ThC101, ThC582, ThC583, ThC585, FC652). Their values are consistently lower than male to female ratios measured within each of the studies, suggesting than in common with other STDs male to female transmission may be more efficient than vice versa.

Finally, some comments about sexual behaviour studies. There was a whole oral session devoted to the studies of adolescent behaviour (Saturday C10) and, in common with the findings of the pilot phase National Study of Sexual Attitudes and Lifestyles in this country (SC1 10), it is clear that it is the youngest age groups who are the most sexually active in terms of rates of partner change. Furthermore, adolescents are also vulnerable because of the emergence of crack in the United States, which may result in young women using crack and prostitution as a source of funding their drug habit. Crack results in enhanced sexual interest and increasing number of partners, and this, in itself, is fuelling the emergence of traditional STDs, particularly in inner city deprived areas in the United States, with the re-emergence of heterosexual syphilis and gonorrhoea.

\section{Natural history of progression of HIV infection to AIDS-Richard Keenlyside}

There were several reports of studies of the natural history of progression of HIV infection. The San
Francisco Cohort of homosexual men showed that AIDS had developed in $53 \%$ of the 489 men within 11 years. Of 341 participants men with an estimated date of seroconversion before $1981 ; 66(19 \%)$ had no signs or symptoms of HIV infection and approximately half had maintained a CD4 count of more than 500 (ThC33).

Among men in the SF General Hospital Cohort, followed now for 5 years, $30 \%$ had progressed to AIDS and $54 \%$ to AIDS or ARC and in Sydney, Australia a cohort of 1057 has been followed for over 5 years and $26 \%$ have now progressed to AIDS (ThC619).

In transfusion associated cases in Mexico $20 \%$ progressed to AIDS within 12 months of infection, $50 \%$ after 2 years and $75 \%$ after 3 years (ThC635). However among haemophiliacs with known dates of seroconversion followed at the Royal Free Hospital the calculated cumulative incidence after 10 years was $49 \%$ for AIDS and $85 \%$ for symptoms (ThC641). These cohort studies suggest a similar rate of progression of the infection in groups in northern countries. However, this does not appear to be the case in countries in Africa. Among Nairobi prostitutes with known duration of infection the median time of progression to stage 4 disease was significantly faster, namely 3 years (ThC37). The differences may reflect gender, geographic location, socio-economic or health status, or perhaps more importantly a greater burden of cofactors such as other communicable diseases.

\section{Kaposi's sarcoma ( $K S$ )}

In the European Collaborative Kaposi's Sarcoma Study the percentage of reported KS cases among homosexuals, heterosexuals and IVDUs has been declining over time (ThC632). It is not clear if this is explained by a bias in reporting, changes in diagnostic practices or decrease in exposure to a possibly sexually transmitted cofactor.

There was much interest in the evidence for a cofactor for Kaposi's sarcoma that might be independently sexually transmitted (ThC631). Several investigators suggested that cofactors for $\mathrm{KS}$ may be transmitted by sexual intercourse or by oral anal contact. A case control study in Vancouver comparing patients with $\mathrm{KS}$ and those with opportunistic infections showed that persons with $\mathrm{KS}$ had more sexual partners, used nitrites more often and admitted to more oral anal contact (ThC630).

Several studies reported that in comparison to other persons with AIDS those with KS had shorter times from seroconversion to AIDS and longer survival times after diagnosis. These findings are consistent with $\mathrm{KS}$ developing at an earlier degree of immunodeficiency and borne out by findings that men with KS tend to have higher levels of CD4 cells at the time of diagnosis (ThC261). 
Other cofactors and determinants of progression to AIDS

Smoking-In the Multicenter AIDS cohort study (MACS) study in the US a dose dependent relationship was found between levels of $\mathrm{CD} 4$ cells and smoking before and after seroconversion. Levels were higher in smokers than non smokers (ThC675). Genetic compostions. Certain HLA types were more common among HIV infected homosexual men in one cohort who were asymptomatic after 5 years or more of follow-up. The authors suggested that HLA may influence disease progression (ThC676).

Surveillance trends in AIDS incidence, survival and mortality

Two important and worrisome facts came from North America: (1) Racial and ethnic minorities continue to have high rates of infection. (2) Young people in many studies have significant rates of infection. For example studies of homeless young people in four states revealed an overall seroprevalence of $4.3 \%$, and a rate that increased with age (FC47). Surveillance in STD clinics in the US reveals an overall seroprevalence of $2.5 \%$ in 115,989 persons tested. In heterosexual males it is $2.0 \%$ and females $-0.8 \%$ (FC44).

Several investigators in the US have reported a levelling off of AIDS incidence and mortality and improved survival among white homosexual and bisexual men (FC41). This was suggested by a study of AIDS incidence, deaths and survival in homosexual men in the US between 1984-88 from national surveillance data. There was a slowing of increase of AIDS in 1986. The percentage surviving at least 12 months increased from $40 \%$ to $56 \%$. Survival figures were better in men diagnosed in the highly endemic areas, NYC, LA, SF suggesting that advances in care may have postponed AIDS deaths (FC687).

Many countries also reported an increase of HIV related mortality in men and women under the age of 49 years as well as among children. All reflected increased rates of premature death and years of potential life lost due to AIDS and HIV infection. The United States national surveillance data reveal AIDS to be an increasingly important cause of death in 20-24 years. It emerges as a leading cause of death in American women of reproductive age-especially black women and in the North Eastern states (ThC744). This is even more the case in Africa where for example in Abijan on the Ivory Coast-AIDS and AIDS related conditions are the leading causes of adult deaths and years of potential life lost (FC103).

\section{Drug users-epidemiology-Graham Hart}

The message from the United States was fairly promising. Prevalence of HIV infection in IV drug users seems to be levelling out for the most part, and in New York there was even a reduced incidence of
AIDS, a peak having been reached in 1988 (ThC707). Unfortunately, in six South Eastern states there was a raised incidence of AIDS amongst heterosexual IDUs and their sexual partners (ThC706). A massive study of HIV infection in 16941 IDUs at 65 sentinel drug treatment clinics in 27 metropolitan areas reported prevalences ranging from $0 \%$ to $47.7 \%$, with the highest being found in the North East of the country as compared to $2.5 \%$ for the rest of the US as a whole (FC551).

It has been suggested that the reasons for a constant prevalence of just over $11 \%$ since 1987 in San Francisco were the reduction in needle-sharing, generally safer needle use, and the use of bleach by those who continue to share (FC106). Moss, reporting from the same city, confirmed that the infection rate has continued to fall, but noted that new infections were likely to be related to sexual risk amongst IDUs; following 604 drug users over the period 1985-90 who were seen twice, 15 seroconverted, giving an incidence of $1.9 \%$ per person year. Risk factors for seroconversion included reporting five or more sexual partners in the last year, use of crack cocaine, shooting gallery use and cocaine injection (FC553).

Whilst many of the American studies described variation between regions, one reported substantial differences in a discrete geographical area. Rates varied in methadone treatment populations in and around Boston acording to location; 30 miles outside the city it was $4 \%$, at a privately owned centre it was $14 \%$, but in the inner city (serving a predominantly black population) it was $31 \%$. Much of the difference was accounted for by reference to ethnicity and unequal access to treatment and prevention services (FC558). By way of contrast, IDUs in Minnesota have access to low threshold methadone maintenance programmes and needles and syringes are available without prescription, and here prevalence has remained consistently low, and was $2 \cdot 2 \%$ in 1989 (FC559).

Although we are sadly not surprised at a prevalence of $47 \%$ in New Jersey amongst injectors, particularly of cocaine and speedballs (cocaine/ heroin mix) (FC561), it is surprising to see a 7\% prevalence amongst non-injectors in the city; Patel et al (FC560) suggest sexual risk as a likely cause of infection amongst this group of non-injecting drug users.

Reports from Europe were also tentatively optimistic, with no major increases reported in Antwerp (4\%) (FC618), Berlin (17\%) (FC625) and, Athens (1.1\%) (FC627); in London, there has been no increase in infection amongst attenders at a needle-exchange over an 18 month period $(2.4 \%)$ (SC745). Two Italian studies, from Verona and Turin found reductions in the prevalence of antiHIV, and maintained that this was associated with 
the availability of methadone treatment and health education campaigns (FC633; FC634).

Until 1987 there were no identified cases of HIV infection among IDUs in Poland, but by $198911.2 \%$ of 5351 drug users in treatment were antibody positive; in 1990 the prevalence was $11.9 \%$ (FC640).

A report from Spain found an overall prevalence in 1233 IDUs from 83 treatment centres of $40 \%$, although this varied by region and city (SC559). There was confirmation of the $69 \%$ prevalence previously reported in Barcelona (FC643), although in Northern Spain generally there has been a reduction in the incidence of new infections between 1984 and 1989 (FC644).

\section{Clinical aspects A-Sarah Gill}

\section{Management of Pneumocystis carinii pneumonia}

Treatment: Many centres have been using inhaled pentamidine in mild to moderate PCP and Montgomery presented the final details of a study in which 379 patients had been randomised to receive either aerosolised pentamidine $600 \mathrm{mg}$ daily via a respirgard II nebuliser or cotrimoxazole $15 \mathrm{mg} / \mathrm{kg} /$ day intravenously (ThB395). This study showed that there was a statistically significant difference in the duration of treatment required with aerosolised pentamidine (that is, longer-time for response) compared with cotrimoxazole. However, patients receiving aerosolised pentamidine had significantly fewer side effects (rashes, raised transaminases and drug fever). So the conclusions are, that aerosolised pentamidine is probably as effective as cotrimoxazole for the treatment of acute mild to moderate PCP and more likely to be tolerated. However, it should be reserved for patients who have less severe hypoxia as the initial response is often slower.

The Californian Collaborative Treatment Group presented a randomised controlled trial of the use of corticosteroids in acute PCP, (SB17). The aim was to establish whether the early use of corticosteroids (within 36 hours of starting treatment) prevented oxygenation failure and reduced toxicity. Three hundred and thirty three patients were enrolled, and randomised to receive either standard therapy alone or standard therapy plus prednisolone $40 \mathrm{mg}$ bd for 5 days, reducing to $40 \mathrm{mg}$ daily for 5 days and then $20 \mathrm{mg}$ daily to the end of therapy. The endpoints were death, ventilatory failure (the need for mechanical ventilation), oxygenation failure (the need for further oxygen administration) or dose limiting toxicity. The conclusions were that the early addition of steroids reduced oxygenation failure, ventilatory failure and death. This study was based on the use of corticosteroids within 36 hours of presentation and there was no controlled data presented on the late use of corticosteroids or their use as salvage therapy.
PCP prophylaxis

In spite of several studies there is no clear answer as to which is the best drug or combination. Montgomery presented data on aerosolised pentamidine from the San Francisco Community Group Study. ${ }^{1}$ Four hundred and eight patients at 12 treatment centres were randomised to receive $30 \mathrm{mg}$ once every 2 weeks, $150 \mathrm{mg}$ once every 2 weeks or $300 \mathrm{mg}$ once a month. Eighteen months after randomisation only eight episodes of PCP occurred in the $300 \mathrm{mg}$ arm versus 22 in the $30 \mathrm{mg}$ arm. There were 16 episodes in the $150 \mathrm{mg}$ arm but this was not significantly different from the high dose arm.

\section{$M A I$ infections}

A study of therapy in Mycobacterium avium intracellular (MAI) infection, with intravenous amikacin together with a combination of clofazimine, rifampicin, ethambutol and ciprofloxacin showed that 19 of the 20 studied patients had a favourable clinical and microbiological response (ThB517). There were severe side effects as a result of ototoxicity in three patients presumed to be due to amikacin. Before 12 weeks most patients experienced an increase in weight and a reduction in fever. Beyond 12 weeks although patients continued on treatment the weight started to fall again and the fevers returned. Another study looked at rifabutin (ansamycin), in 22 patients with AIDS and other opportunistic infections who were being prescribed this drug as an anti HIV agent (ThB518). These patients were compard retrospectively to 184 similar patients, and there seemed to be a trend towards more MAI free survival in the rifabutin treated group as long as they had treatment for more than 30 days.

\section{$H I V$ in health care workers}

Bell from the Centers for Disease Control presented 14 studies in which 1962 health care workers reported a total of 8000 exposures to HIV (FC33). There were six seroconversions in total and the relative risk of seroconversion per needlestick injury was estimated to be $0.31 \%$. Data were presented on how these injuries occur. Thirty one per cent of them were due to surgeons holding tissue with fingers while suturing, $10 \%$ were the result of surgeons holding needles and tying, $6 \%$ from sharp passing in theatre and $46 \%$ from other injuries (FC34). Chemoprophylaxis using zidovudine ( $\mathrm{ddI}$ and $\mathrm{ddC}$ ) on the grounds that they probably prevent viral replication or $\alpha$ interferon, soluble CD4 and on the immunoglobulins which may prevent entry have been considered, but only zidovudine has been used in practice. San Francisco General Hospital have started a protocol (FC35) in which they offer health care workers (HCW) who have had a needlestick injury zidovudine for 4 weeks. The recipients are instructed on how locally to decontaminate the exposure. There 
is a needlestick hotline and they receive expert counselling and advice so that they can reach a decision about whether or not to take zidovudine. If they do opt for zidovudine this is preferably administered within an hour. All exposed persons are followed up every 2 weeks during treatment, at 6 weeks, 3 months and 6 months after exposure. This protocol has increased referrals by $168 \%, 63 \%$ of HCWS reported needlestick injuries, $10 \%$ transcutaneous injuries and the rest mucus membrane exposure. Twenty eight persons have had eligible exposures and 16 have opted for zidovudine which they have received within an hour. In five cases the donor was found to be HIV negative so treatment was discontinued. In six cases there were significant side effects so treatment was discontinued and in a further case treatment was discontinued for other reasons so only four completed treatment. However, all of these exposures subsequently turned out to be HIV negative after the complete follow up.

\section{Clinical aspects B-Melinda Tenant-Flowers Cerebral toxoplasmosis}

Two studies from France (ThB476, ThB481) linked the incidence of this infection with low CD4 counts. Between $78-84 \%$ of their patients at the time of diagnosis of cerebral toxoplasmosis had CD4 counts below $100 \mathrm{~mm}^{3}$ and $79 \%$ below $150 \mathrm{~mm}^{3} 3$ months prior to diagnosis. This raises the question as to the efficacy of primary prophylaxis against toxoplasmosis in patients with low CD4 counts particularly in countries where the prevalence of infection is high. A placebo controlled study with pyrimethamine is underway in France.

The main treatment news came out of the California Collaborative Trials Group Study of the efficacy of clindamycin and pyrimethamine versus sulphadiazine and pyrimethamine for induction treatment (SB20). Although quite a small study, it showed that the clindamycin regime was as efficacious as the standard sulphadiazine treatment and there was no marked difference in toxicity between the two groups. Clindamycin has been used as second line treatment for cerebral toxoplasmosis in patients sensitive to sulphanamides for some time and this study is an important confirmation of its efficacy. An Australian group looked at sulphadiazine desensitisation in a group of patients allergic to sulphadiazine and clindamycin (ThB480). Sixteen patients were desensitised using gradually increasing increments of oral sulphadiazine 3 hourly over 5 days, this was successful in 10 patients.

The relapse rate if maintenance treatment or secondary prophylaxis is not given is between 37$86 \%$. Different maintenance regimes were presented. However, all of these studies involved small numbers of patients and the results should be interpreted with caution. A study of 27 patients from Spain (ThB483) involved the use of intermittent sulphadiazine and pyrimethamine versus clindamycin and pyrimethamine, the drugs were given orally on 2 days/week. There were no relapses in the sulphadiazine and pyrimethamine group compared to $40 \%$ in the clindamycin group. A study from Holland looked at the use of various maintenance schedules including pyrimethamine alone (NEU-3) and found that in a higher dose group receiving $50 \mathrm{mg} /$ day there were fewer relapses but more haematological side effects. A study from the USA looked at the efficacy of PCP prophylaxis regimes as secondary prophylaxis against toxoplasmosis (ThB482). Four of the 97 patients who received double strength cotrimoxazole tablets developed toxoplasmosis compared with 5 of 86 who received inhaled pentamidine.

\section{Cryptosporidiosis}

The severity of the disease appears to be linked to the degree of immunosuppression. A study which looked at eight patients who cleared their infections spontaneously, showed that their mean CD4 counts were higher $287( \pm 246)$ than those of 18 patients with persistent infection $64( \pm 12) \mathrm{p}<0.05$ (ThB519). There were an equal number of patients in both groups who were on zidovudine.

There has been very little progress in the treatment of this condition. A trial of diclazuril (ThB520) an agent active against Eimeria sp (a protozoan similar to crytosporidiosis which infects chickens) was presented. A complete response was obtained in two out of 30 patients and a partial response in $50 \%$. All responses were in patients on higher doses of the drug (that is, between 400 and $600 \mathrm{mg} /$ day). Hyperimmune Bovine Colostrum was not shown to be efficacious (ThB521).

\section{Obstetric and paediatric aspects of HIV infection}

The transmission risk of HIV from mother to child is reported to be between 20 to $45 \%$ and a paper from France (ThC44) identified three main maternal factors associated with an increased risk of transmission: a CD4 count below 150, P24 antigen positivity, and a high viral load. Pregnancy is not associated with an increased risk of progression of HIV disease but is associated with maternal morbidity. A Russian group (ThC48) reported eight supposed cases involving transmission from infant to mother after breast feeding. Seven of the infants had aphthous ulcers, and two of the mothers had cracked nipples and no other risk factors for HIV infection.

There is no single reliable test available for the diagnosis of paediatric HIV infection. Tests that are currently in use include PCR (FB446) which has high sensitivity but is liable to contamination, IgA to envelope proteins (ThC46) which becomes positive early at 5 weeks and Beta 2 Microglobulin (FB444) which becomes elevated at 6 months. 
There has now been a phase 1 trial of AZT in pregnancy given to 10 women in the last trimester (FB17). It freely crosses the placenta and it remains to be seen whether it prevents transmission. Phase II trials of AZT in children from the USA (FB18) and Spain (FB20) both demonstrated clinical improvement on treatment. Side effects occurred in 15 to $19 \%$ of patients, including insomnia, vomiting and haematological toxicity. A ddI phase $\mathrm{I} / \mathrm{II}$ trial in 43 children using variable doses (FB22) has also demonstrated clinical improvement, increased CD4 counts, a drop in P24 Ag levels, two children had pancreatitis, two became neutropaenic but there was no neurotoxicity or death directly attributable to ddI.

\section{Neurological aspects-Hadi Manji Crytococcal meningitis}

In San Francisco, there were two studies presented, one which compared amphotericin B and fluconazole in the acute treatment of crytococcal meningitis and the second looked at the question of maintenance treatment.

The first study on acute treatment compared fluconazole given at a dose of $400 \mathrm{mg}$ on day 1 followed by $200 \mathrm{mg}$ daily for 10 weeks, with amphotericin at a dose of at least $0.3 \mathrm{mg} / \mathrm{kg} /$ day IV (with or without 5 Flucytosine) for the same period (SB19). Successful treatment was defined by improvement clinically and with 2 negative CSF cultures. Roughly the same number of people improved on fluconazole $(34 \%)$ and amphotericin $(40 \%)$. The mortality rate was high in both groups $20 \%$ and $17 \%$ respectively. However, the early mortality with fluconazole, $15 \%$ compared to $8 \%$ for amphotericin, is worrying. The other concern is that CSF culture became negative much more rapidly with amphotericin B than with fluconazole and this may relate to the early mortality in the fluconazole group. However, by 10 weeks there was no significant difference between the 2 groups in terms of CSF culture of the organism. The toxicity, as expected, was higher in the amphotericin group.

The maintenance (Aids Clinical Trial GroupACTG 026) trial, compared weekly amphotericin B at a dose of $1 \mathrm{mg} / \mathrm{kg} /$ week IV versus fluconazole $200 \mathrm{mg} /$ day orally (SB19). These patients had all been treated for acute crytococcal infection with amphotericin $B$ and subsequently had two negative CSF cultures. The results show that $2 \%$ of the group on fluconazole relapsed compared to $17 \%$ on amphototericin $B$ and furthermore the toxicity in the fluconazole group was only three out of 106 as compared with $12 / 42$ for amphotericin B. Patients given amphotericin $B$ had to be given the drug by a central line with all its problems and complications.

Holton et al from the University of Southern California presented a poster (ThB461) in San Francisco which looked at the efficacy of intrathecal amphotericin B. They concluded that it offered no extra benefit. In fact four out of 15 patients developed reversible spinal cord problems. At Monterey, Aronow et al from Los Angeles presented data on the use of ketoconazole $200 \mathrm{mg}$ /day as a primary prophylactic agent for crytococcal meningitis (Monterey, NEU-1). This was a retrospective study in AIDS or ARC patients those CD4 counts were less than 400 . Their results suggested that of the group given ketoconazole one out of 229 developed crytococcal meningitis and 10 out of 123 who were not given ketoconazole developed the infection giving an incidence of $8 \%$, which is roughly the expected incidence in the California area. However, this was a retrospective study. Further randomised prospective studies are needed before ketoconazole can be recommended as prophylaxis against crytococcal meningitis.

In summary, fluconazole does seem efficacious in the treatment of acute crytococcal meningitis, it certainly is less toxic than amphotericin B. However, because of the high early mortality and the fact that the organisms took much longer to clear from the CSF patients who are seriously ill and who have features suggestive of a poor outcome (CSF antigen greater than one in 10,000 , India ink smear positive CSF, hyponatraemia and the culture of cryptococcus from excranial sites) should perhaps still be on amphotericin in the first instance and maybe change to fluconazole for maintenance therapy.

\section{Effect of sterotactic brain biopsy in AIDS}

Levy and his colleagues from Chicago prospectively looked at the value of stereotactic biopsies in AIDS patients (Monterey, NEU-7). These were CT guided biopsies in the main although a number were MRI guided. They performed 34 biopsies on patients who presented with solitary lesions who failed or had an equivocal response to antitoxoplasmosis treatment. Nine turned out to be toxoplasma, nine CNS lymphoma and nine PML, HIV encephalitis was diagnosed in three and single diagnoses of malignant melanoma, cryptococcoma, and MAI were also made. Two were non-diagnostic giving a diagnostic efficacy of $93 \%$. With regards to complications, three patients suffered haemorrhages and in one there was a residual hemiparesis. Thete were no deaths. In the right hands, stereotactic biopsy seems to be both safe and effective in obtaining a diagnosis. ${ }^{2}$

\section{Primary central nervous system lymphoma}

Rosenblum and his colleagues from Chicago presented data at Monterey (NEU-9) on the natural history and response to radiotherapy in primary CNS lymphoma. They looked at 55 patients, $90 \%$ of whom had the diagnosis made on stereotactic biopsy with no significant mortality or morbidity. Twenty nine patients were given radiotherapy, with 4000 rads 
over a period of 3 weeks. Seventy six per cent of patients improved clinically and $70 \%$ improved radiologically; $10 \%$ deteriorated clinically and $9 \%$ radiologically. With radiotherapy the mean survival was 134 days compared with $\mathbf{4 2}$ days in the patients who had no radiotherapy. Nearly $90 \%$ of the patients given radiotherapy died of an opportunistic infection whereas $77 \%$ of the group not given radiotherapy died of tumour progression. A drawback of this paper is that we are not told whether the 2 groups were comparable in terms of general physical condition. Patients suspected of having a lymphoma should undergo early biopsy and radiotherapy. As we get better at treating opportunistic infections hopefully the mean survival in this group of patients will be increased.

\section{Progressive multifocal leucoencephalopathy}

Galli et al from Milan looked at the autopsy results of 385 AIDS patients of whom $31(8 \%)$ had PML. The usually quoted incidence in AIDS is $2-4 \%$ (ThB449). Interestingly 26 of this group were intravenous drug users. The patients presented with a wide range of neurological symptoms including focal weakness, gait disturbance, altered mental status, visual loss, cranial nerve palsy and dysarthria and PML is a diagnosis to be borne in mind with any patient presenting with neurological illnesses. Furthermore, $50 \%$ had other additional CNS pathologies which included HIV encephalitis, opportunistic infections and lymphoma demonstrating the problem of multiple pathology in AIDS.

Weber et al from Germany were able to show evidence of the JC virus in the CSF using PCR in patients with PML but could show no evidence of the virus in 32 controls (Monterey, NEU-2). This may become an important non-invasive diagnostic tool in the future.

\section{Myopathy, HIV, zidovudine}

Myopathy in HIV is a rather heterogenous histological group of conditions with biopsies showing an inflammatory polymyositis, a non-inflammatory destructive myopathy, a nemaline rod myopathy or a mixture of all these different sorts. To date there has been no clinical, biochemical, electrophysiological or histological way of differentiating between a myopathy due to HIV or a myopathy due to zidovudine. Some people actually doubt the existence of the latter. There were two presentations in Monterey on myopathy. The first was by Dalakas from Bethesda who looked at 23 patients with myopathy, five of whom who had never been on zidovudine (Monterey, BS-8). All these patients presented with proximal myopathy, a raised $C K$ and a myopathic EMG. Both zidovudine treated and untreated patients had evidence of inflammation on their biopsies consisting of CD8 positive $T$ cells and macrophages gathering round muscle fibres which had expressed the $\mathrm{MHCl}$ antigen. In addition, the group who had been on zidovudine showed ragged red fibres and abnormal mitochondria with paracrystalline inclusion bodies. Two patients who stopped zidovudine and had subsequent biopsies, showed an improvement in the number of ragged red fibres and abnormal mitochondria but they still maintained the degree of inflammation as compared with the first biopsy; seven out of 18 patients improved on stopping zidovudine and four out of 18 improved with steroids. In the group that had never been given zidovudine two responded to steroids, one patient died and one patient improved when given zidovudine. The authors concluded that the myopathy in the patients who had been on zidovudine was due to a combination of the inflammatory response which is steroid responsive together with zidovudine acting as a reversible mitochondrial toxin.

A study from Simpson et al at Mount Sinai Hospital (Monterey, NEU-15) of 35 cases of HIV associated myopathy of whom 20 had been on zidovudine and 15 who had not, found mitochondrial abnormalities in both the zidovudine and the nonzidovudine groups, but very little evidence of ragged red fibres. As far as treatment was concerned 10 patients were withdrawn from zidovudine and in eight there was no benefit, eight out of nine with progressive weakness responded to steroids.

In summary, the relationship between myopathy, zidovudine and HIV is still not clear. Prospective trials stopping zidovudine and using steroids are needed.

\section{CMV retinitis}

There were a number of posters which looked at the efficacy of both foscarnet and ganciclovir in the treatment of CMV retinitis. Moyle et al from Westminster Hospital, compared ganciclovir and foscarnet in the acute treatment of CMV retinitis (FB95). There were 22 patients in each group-18 in the ganciclovir $(5 \mathrm{mg} / \mathrm{kg} / \mathrm{bd})$ and 12 in the foscarnet $(200 \mathrm{mg} / \mathrm{kg} /$ day $)$ group, improved or stabilised. However, six patients required a prolonged course of induction treatment with foscarnet. Side effects included neutropenia in the ganciclovir group and in the foscarnet group complications were due to raised urea, hypocalaemia and penile ulceration. For maintenance patients were given either drug on a five out of 7 day regime, ganciclovir $5 \mathrm{mg} / \mathrm{kg} /$ day, or foscarnet $130 \mathrm{mg} / \mathrm{kg} /$ day. The reactivation rate was similar in both groups of patients at $25 \%$. However, central line sepsis was much higher in the ganciclovir group postulated to be due to neutrophil dysfunction.

A poster (Hardy et al, FB92) in San Francisco looked at the safety and efficacy of ganciclovir versus ganciclovir plus granulocyte-macrophage colony- 
stimulating factor (GMCSF). Although the occurrence of neutropenia was less, the incidence of line sepsis was much the same. Patients given the combination therapy had an increased incidence of severe myalgias and thrombocytopenia.

\section{Cognitive dysfunction in HIV positive patients}

Several large studies suggest that there is no evidence of significant neuropsychiatric impairment in asymptomatic HIV positive patients, compared with HIV negative patients in the same high risk categories. ${ }^{3-5}$ All the studies have a number of problems including those of sampling, patient selection (many studies are composed of white, highly educated, highly motivated homosexual males and it is difficult to extrapolate any results from this group of patients to other HIV groups), the neurophysiological tests used tend to differ between studies; the criteria used for defining abnormalities are variable and in a number of studies no account is taken of confounding variables such as mood, alcohol and drug abuse.

Finally, I mention a study by Baum and Wilki from Miami (PSY9) who considered vitamin $B_{12}$ levels and cognitive function in HIV positive patients. They looked at $100 \mathrm{HIV}$ positive asymptomatic patients who were on no drug therapy and 50 seronegative controls. $B_{12}$ levels were measured on more than one occasion and patients underwent clinical examination and psychometric testing. Interestingly $20 \%$ of the HIV positive group had $B_{12}$ deficiencies $\left(B_{12}\right.$ levels of less than $200 \mathrm{pg} / \mathrm{ml}$ ). All these patients had a normal MCV, normal thiamine and folate levels. A further, $14 \%$ of the HIV positive group had borderline $B_{12}$ (between 200 and 250). It was postulated that the low $B_{12}$ levels may reflect a subclinical malabsorption syndrome.

The results of neuropsychometric testing, (using paired associates mean reaction time, the visual figure and the mean reaction time for the Sternberg short term memory paradigm scanning task) suggest there was a difference in the group who were HIV + and low on vitamin $B_{12}$ compared with HIV + group with normal $B_{12}$. Those patients with low $B_{12}$ levels given supplements improved on some of the tests after 6 months whereas the patients who for various reasons were not prescribed $B_{12}$ continued to do poorly. This is an area which needs further investigation.

\section{Zidovudine-Ian Weller}

Four zidovudine trials were carried out by the AIDS Clinical Trial Group (ACTG) in USA-016, 019, 02 and 010 are reviewed. Firstly, protocol 016 was a placebo-controlled trial in patients with early symptomatic disease, that is a CD4 cell count between $200-800$, and one or two HIV-related features including oral candida, oral hairy leukoplakia, single dermatomal herpes zoster, diarrhoea, weight loss and seborrhoic dermatitis. ${ }^{\circ}$ The dose used in this study was $1200 \mathrm{mg}$. The important thing about this study is that it does provide some evidence in addition to the first placebo controlled trial in advanced disease that zidovudine delays progression in early symptomatic disease. The endpoints were AIDS or severe $A R C$ and patients were stratified according to their CD4 cell count. The main results of the study apply to the 513 individuals with CD4 counts between 200 and 500 at entry. There were a total of 40 progressions overall, 34 in the placebo arm and 12 in the zidovudine arm and this was statistically significant. The results of this trial on clinical practice are that it will make physicians feel more confident about giving zidovudine in patients with early symptomatic disease.

ACTG 019 was a trial in asymptomatic patients. This study included over 3,200 patients recruited between July 87 and July 89 . There were three arms to the trial, $0.5 \mathrm{~g}$ versus $1.5 \mathrm{~g}$ versus placebo. The endpoints were either severe ARC or AIDS and patients again were stratified according to their CD4 counts. The results now published refer to the 1338 patients with CD4 counts $<500$ at entry. ${ }^{7}$ The mean follow-up was just over one year with a range of four months to two years. The progression rate to AIDS and advanced ARC was approximately halved from $7 \cdot 6 / 100$ patient years in the placebo group, to 3.6 and 4.3 in the low and high dose groups respectively. However, only a minority of individuals actually benefited in the mean follow-up of 55 weeks. Severe anaemia occurred in $6.3 \%$ of the high dose group compared to $1.1 \%$ in the low dose group and the latter was not significantly different from the incidence in the placebo arm. On the basis of these results this trial was interrupted and all patients with CD4 counts $<500$ were offered open zidovudine. The patients with CD4 counts $>500$ are still on study.

ACTG 002, was a study of 524 patients with first attack pneumocystis who were randomised to either the full dose, zidovudine $(1500 \mathrm{mg} /$ day $)$ versus $600 \mathrm{mg}$ daily (patients in this arm were given $1200 \mathrm{mg}$ daily for the first month). ${ }^{8}$ The median follow-up was for 26 months. The number of new opportunistic infections that occurred on follow-up, the time to the second infection, the increase in CD4 counts and the decrease in P24 antigen were all similar in both groups. However, there was a survival difference, that is, a bulge in the survival curve in favour of the low dose. There was also a difference in terms of toxicity with far more haematological events in the high dose arm. Because of dose reductions and interruptions in therapy because of toxicity, the high dose arm received a median cumulative zidovudine dose of only $204.5 \mathrm{~g}$ versus $169.1 \mathrm{~g}$ in the low dose arm. The reason for the apparent difference in survival is unclear. A significantly greater proportion of patients in the low dose group received 
pneumocystis prophylaxis earlier in the study although the authors felt that this did not account for the difference in survival.

The results of 019 and 002 are now being used to support the low dose $500 \mathrm{mg}$ in routine clinical practice. However, it should be appreciated that these trials compared 500 and $600 \mathrm{mg}$ to $1500 \mathrm{mg}$. In Europe $1000 \mathrm{mg}$ has been a standard dose and is being employed in the current trials in asymptomatic individuals. Whether $500 \mathrm{mg}$ is better than $1000 \mathrm{mg}$ is unknown as is the effect of low doses on prevention of CNS disease and the rate of appearance of resistant viral strains.

ACTG 010 was a study designed to examine whether acyclovir would potentiate the antiviral action of zidovudine. Patients were randomised to $300 \mathrm{mg} /$ day, $600 \mathrm{mg} /$ day or $1500 \mathrm{mg} /$ day of zidovudine together with $800 \mathrm{mg} 4$ hourly of acyclovir. It has since been shown that acyclovir does not potentiate the anti-HIV effect of zidovudine and what this study therefore becomes is a zidovudine dosecomparison study using HIV plasma viraemia or virus titre in plasma and P24 antigen levels as endpoints. The $300 \mathrm{mg}$ dose of zidovudine appeared to have similar virological effects (as measured by effect on P24 antigenaemia and plasma virus titres) to doses of $600 \mathrm{mg}$. This interesting observation needs to be confirmed in larger numbers of patients before it is allowed to affect clinical practice. ${ }^{9}$

The genetic mechanism of resistance was described $^{1011}$ in 1989. It appears to be due to combinations of four restricted mutations of the reverse transcriptase gene, generally speaking the more mutations the more resistant the virus is. Viral constructs with these mutations are also resistant, and most importantly these mutations are not found in wild type viruses.

In a workshop on resistance some groups described viruses which only have one of these mutations and yet show very high levels of resistance and questions were raised as to whether other mutations of reverse transciptase might be contributing. As far as the prevalence is concerned the majority of patients with symptomatic disease at the end of one year will have resistant viruses and in a proportion of them this may reach quite high levels. In high risk asymptomatic patients who are antigenaemic, with low CD4 counts, a study of 18 patients from Amsterdam showed that the majority by two years have resistant viruses, but the resistance is at a lower level than that seen in symptomatic patients. ${ }^{12}$ Finally, in asymptomatic individuals around $20-30 \%$ of patients will have resistant viruses by about 18 months but again at a very much lower level than in symptomatic patients (SB82). So it appears that in asymptomatic patients these resistant mutants become dominant in a small proportion of individuals and they are retarded in terms of their rate of emergence. There are no data which clearly correlate clinical deterioration and the appearance of resistant virus. We still do not know how long the virus persists when therapy is stopped. A number of different assays are being used to assess this phenomenon and it is likely that some of the different results that are being found are because of differences in assays. If resistance is an important thing to monitor then we need a simple test. Other concerns include possible transmission of these resistant viruses from a patient on zidovudine to a partner not on zidovudine, and the possible association between the emergence of resistance and dose of zidovudine. Finally, we need to know whether combination treatments prevent or delay the emergence of resistant strains.

CD4 lymphocyte phenotyping is a very important area which we have to address if we are to use CD4 counts as markers for early therapeutic intervention. CD4 lymphocyte phenotyping has been moved very rapidly into the clinical arena when it was still a research laboratory tool. It is a very variable parameter and depends on the patient's condition, exercise, drugs, and intercurrent infections. ${ }^{13}$ There is a diurnal variation with the lowest level in a normal waking/sleeping pattern in the morning and the highest in the evening, in immunocompetent individuals. There is also a seasonal variation with higher levels in summer than in winter, which is important if one is monitoring trends over time. The value depends on how the sample is handled and whether it is kept at an ambient temperature. The practice of high risk batching leaving the high risk samples to the end of the day can lead to a falsely low absolute CD4 cell count. Flow cytometry is the best method for $\mathrm{CD} 4$ counting as much larger numbers of cells can be counted but some units are still using microscopy. Whole blood techniques generally give better results than those techniques which rely on separating lymphocytes. Issues of quality control and local normal ranges in different groups are all important.

The aims of combination chemotherapy are to reduce zidovudine dose by using another antiviral in combination to retain or enhance efficacy, reduce toxicity and hopefully prevent or delay the emergence of resistant viral strains. Ideally not another dideoxynucleoside but a drug inhibiting another site of the viral replicative cycle is required. However, these are not yet available. ACTG 047 , is a study to compare alternating and intermittent regimes of zidovudine $1200 \mathrm{mg}$ daily and ddC in ARC and AIDS. Two doses of ddC are being used low $(0.01$ $\mathrm{mg} / \mathrm{kg}$ four hourly) and high ( $0.03 \mathrm{mg}$ four hourly) (ThB23). The results presented were very preliminary and look promising. There is a decrease in haematological toxicity and peripheral neuropathy appearing in the combination arms compared to either drug alone and increases in $\mathrm{CD} 4$ counts and 
decreases in P24 antigen levels. There was a hint that the monthly alternating regimen was better than the weekly. Other trials are beginning or are in progress to study the effect of concomitant administration of ddC and other dideoxynucleosides such as ddI in combination with zidovudine.

The other combination that looked promising was zidovudine and alpha interferon in patients with CD4 cell counts $>500$ (ThB22). The three arms to this study were firstly zidovudine $200 \mathrm{mg}$ four hourly, secondly interferon $5 \times 10^{6} \mathrm{iu}$ and finally zidovudine $100 \mathrm{mg}$ four hourly combined with interferon $1 \times 10^{6}$ iu with the possibility of increasing the interferon dose. The study seems to be showing that the best combination is $600 \mathrm{mg}$ daily of zidovudine with low dose interferon. There was a suggestion that the decrease in P24 in the combination arm was much better than either drug alone.

Finally, just to summarise on other antivirals, there was nothing new presented on ddI. D4T, another nucleoside analogue, was shown in a phase I study to decrease P24. Unfortunately its use is associated with peripheral neuropathy, anaemia, and abnormal liver function tests but lower doses are being explored in further trials. Recombinant CD4 has been very disappointing so far in terms of its effect on surrogate namely P24 and CD4 counts. There is no CSF penetration and secondly patients have antibodies to soluble $\mathrm{CD} 4$ which may well block the activity of the drug so although it worked in vitro extremely well it may not work in vivo (ThB85). It also has a very short half life and as a result CD4 has been coupled as a chimaera to the heavy chain of IgG rCD4-1gG ("Immunoadhesin"). The results of preliminary Phase I dose escalating studies have again been disappointing but higher doses are being studied.

1 Leoung GS, Feigal Jr DW, Montgomery AB, et al. County Community Consortium. Aerosolised Pentamidine for prophylaxis against pneumocystis carinii pneumonia. $N$ Engl $J$ Med 1990;323:769-75.

2 Galgiani JW. Fluconazole, a new antifungal agent. Editorial. Ann Intern Med 1990;113:177-9.

3 Janssen RS, Saykin AJ, Cannon L, et al. Neurological and Neuropsychological manifestations of HIV-1 infection. Ann Neurol 1989;26:601-11.

4 McArthur JC, Cohen B, Selnes O, et al. Low prevalence of neurological and neuropsychological abnormalities in otherwise healthy HIV-1 infected individuals: results from the Multicenter AIDS Cohort Study. Ann Neurol 1989;26: 601-11.

5 Selnes OA, McArthur JC, Gordon B, et al. HIV-1 infection. No evidence of cognitive decline during the asymptomatic stages. Neurology 1990;40:204-8.

6 Fischl MA, Richman DD, Hansen $\mathrm{N}$, et al. The safety and efficacy of zidovudine (AZT) in the treatment of subjects with mildly symptomatic human immunodeficiency virus type 1 (HIV) infection. A double-blind, placebo-controlled trial. Ann Intern Med 1990;112:727-37.

7 Volberding PA, Lagakos SW, Koch MA, et al. Zidovudine in asymptomatic human immunodeficiency virus infection. A controlled trial in persons with fewer than $500 \mathrm{CD} 4$-positive cells per cubic millimetre. N Engl J Med 1990;322:941-9.

8 Fischl MA, Parker CB, Pettinelli C, et al. A randomised controlled trial of a reduced daily dose of zidovudine in patients with the acquired immunodeficiency syndrome. $N$ Engl J Med 1990;323:1009-14.

9 Collier AC, Bozzette S, Coombs RW, et al. A pilot study of lowdose zidovudine in human immunodeficiency virus infection. $N$ Engl J Med 1990;323:1015-21.

10 I arder BA, Darby G, Richman DD. HIV with reduced sensitivity to zidovudine (AZT) isolated during prolonged therapy. Science 1989;243:1731-4.

11 Larder BA, Kemp SD. Multiple mutations in HIV-1 reverse ranscriptase confer high-level resistance to zidovudine (AZT). Science 1989;246:1155-8.

12 Boucher CAB, Tersmette $M$, Lange JMA, et al. Zidovudine sensitivity of human immunodeficiency viruses from high-risk, symptom-free individuals during therapy. Lancet 1990;ii:336, 585-90.

13 Bird AG. Monitoring of lymphocyte subpopulation changes in the assessment of HIV infection. Genitourin Med 1990;66:133-7. 